\title{
Dietary Diversity Practice and its Influencing Factors Among Pregnant Women in Afar Region of Ethiopia: A Cross-sectional Study Design Supplemented by Qualitative Study.
}

Temesgen Gebeyehu Wondmeneh ( $\nabla$ tomigeb2006@gmail.com )

Samara University

Research Article

Keywords: Dietary, diversity, factor, pregnant, women

Posted Date: November 19th, 2021

DOI: https://doi.org/10.21203/rs.3.rs-1080595/v1

License: (9) This work is licensed under a Creative Commons Attribution 4.0 International License.

Read Full License 
1 Dietary diversity practice and its influencing factors among

2 pregnant women in Afar region of Ethiopia: A cross-sectional

3 study design supplemented by qualitative study.

4 Temesgen Gebeyehu Wondmeneh ${ }^{1^{*}}$

5 1. Department of Public health, college of health science, Samara University, Ethiopia.

$6 \quad{ }^{*}$ Correspondent author

$7 \quad$ Temesgen Gebeyehu Wondmeneh

$8 \quad$ Email: tomigeb2006@gmail.com 
Background: Women in low-income countries are frequently malnourished when they become pregnant, and the demands of pregnancy can exacerbate nutritional deficiencies, particularly micronutrient deficiencies, with serious health effects on the fetus. Antenatal nutritional supplements can help to improve birth outcomes and maternal health. As a result, determining the magnitude of dietary diversity and its influencing factors among pregnant women in the pastoral region of Afar, where no study has been conducted, is an essential in order to establish an intervention program in the region.

Method: A mixed study comprising 241 pregnant women and six focus group discussions was conducted from October 1 to November 10, 2018. Participants in the quantitative study were selected by a systematic sampling method, whereas those in the focus group discussions were chosen by using purposive sampling method. The data was collected using pretested questionnaires administered via face-to-face interviews. The relationship between dietary diversity practice and its affecting factors was investigated using logistic regression analysis. The strength of the association was determined by odds ratio with a $95 \%$ confidence interval. Thematic framework was used to analyse the qualitative data. Results: Seventy-three percent of pregnant women had poor dietary diversity. Dietary diversity was higher in younger pregnant women who were under the age of 20 years $(\mathrm{AOR}=5.8$; at $95 \% \mathrm{Cl}: 1.6-13.5)$ and aged between $21-25$ years $(\mathrm{AOR}=3.9$; at 95 percent $\mathrm{Cl}: 1.1-12.2)$ than in older pregnant women with over the age of 30 years. Those participants with a high average family income (above 4500 birr) had a good dietary diversity when compared to those with family income less than 1500 birr ( $A O R=0.1: 95 \%$ $\mathrm{Cl} ; 0.02-0.7)$ and between 1500-3000 birr (AOR=0.05:95\% Cl;0.01-0.2). Pregnant women 
41 who had one antenatal care visit practiced less dietary diversity than those who had four

42 or more (AOR=0.18: 95 percent $\mathrm{Cl} ; 0.04-0.8$ ). Protein-rich foods (meat and eggs),

43 semisolid foods (porridge and cereal soup), milk, fruit (banana) and vegetable (cabbage)

44 were the most commonly avoided foods by pregnant women. These meals were

45 commonly avoided since they produced large babies and were attached to the fetus's

46 body.

47 Conclusion: The majority of study participants had a poor dietary diversity. Pregnant

48 women with a low family income and only one prenatal care visit were less likely than

49 those with a high family income and four or more antenatal care visits to practice dietary

50 diversity, respectively. Most pregnant women avoided high-protein diets, semi-solid

51 foods, milk, vegetable and fruit. Due to the presumptions of producing large fetus and

52 attached to the fetus's body, these foods were avoided.

53 Key words: Dietary, diversity, factor, pregnant, women 
56 Nutrition is critical in reducing pregnancy risks such as maternal and child mortality,

57 intrauterine growth retardation, low birth weight and early births, birth abnormalities, 58 cretinism, poor brain development, and infectious risk. Pregnant women require a diversified diets and increased nutritional intake to cope with the increased demand 60 during pregnancy (1). In a study of low and middle-income countries, women from the

61 Caribbean and Central/South America had higher mean calorie, fat, protein and 62 carbohydrate consumption than women from Africa and Asia. Cereal-based foods 63 constituted the majority of people's diets across the region (2). Based on a study in 64 Eastern Nigeria, around 37\% of respondents avoided certain foods during pregnancy 65 owing to food taboos. Snail and grass-cutter meat were the most common foods to avoid 66 during pregnancy, whereas egg was the most common foods avoiding in children under 67 the age of two-years. Some women thought that consuming snail and grass-cutter meat makes a foetus sluggish and makes a labor more difficult. Food avoidance was not 69 associated with maternal educational status, parity and occupation (3). A study in Kenya 70 showed that cereals were the most consumed food group (99\%), with a mean and 71 standard deviation dietary diversity score of 6.84 and 1.46 , respectively. A 72 minimum of dietary diversity was acquired by the majority of responders (98 73 percent). Only about $2 \%$ of pregnant women did not receive the minimum required 74 amount of dietary diversity. Pregnant women's dietary diversity was influenced by 75 education level, employment status and monthly income (4). A study in Oromia 76 region of Ethiopia among pregnant women revealed that they did not change the amount 
77 and type of foods they consumed to increase nutritional needs during pregnancy. There were a number of taboos related to the consumption of certain food items such as consumption of green leafy vegetables, yogurt, cheese, sugar cane, and green pepper. The taboos were more practiced by older women from rural communities and those with no formal education than those of younger and formal educated mothers, respectively.

82 Fear of weight gain during pregnancy, which is linked to the difficulty of delivering a large baby, was one of the reasons for avoiding these foods (5). According to a study conducted in Wondo Genet district of Southern Ethiopia, most pregnant women's nutrient intakes 
100 Although few studies have been conducted in other parts of Ethiopia, the practice of 101 dietary diversity and its associated determinants among pregnant women was not studied 102 in the context of Afar pastoral region, where sociocultural and economic realities are 103 considerably different. In order to address this knowledge gap, a study was established 104 to assess the magnitude of dietary diversity practiced and its influencing factors among 105 pregnant women residing in Awash seven district, Afar region of Ethiopia.

106

107

108 


\section{Study area}

121 The study was conducted at Awash seven health facility in Afar region of Ethiopia. A large

122 extent of Afar people are a pastoral and follow the Muslim religion. The region's climate

123 is dry and hot, and food shortages occurred on a yearly basis. Aid organizations assisted

124 almost all of the people (under safety net). Cattle, goats, sheep, and camels are the most

125 common livestock used by households. During the dry season, migration of livestock to

126 the major river for pasture and water is common. The Afar social structure is based on

127 descent and affine relationships. The Afar has a patrilineal descent system that assigns

128 a person to a certain clan (the society called mela). Clan members are supposed to share

129 resources and assist one another in the event of an emergency. Cross-cousin marriages

130 are compulsory under Islamic rule. Engagement for marital commitments can start as

131 early as childhood (locally called Absuma). The real wedding usually takes place when

132 girls are in their mid-teens. The boy's father arrives with two camels loaded with butter

133 (locally known as subah), wheat flour, and mat (locally called Senan), and transports the

134 couple to the location where they would build their own home. When a wife becomes

135 pregnant, the husband usually returns her to her family to give birth. Afar people have a 136 strong desire to have as many children as possible. This is because children are viewed 137 as a way of increasing household income by engaging in a variety of activities such as

138 trade, salt caravans, labour migration, and herding (9). Female genital mutilation is a 139 common practiced in the Afar society. Women, men, religious leaders and traditional birth 140 attendants are a key role in the continued practice of female genital mutilation (FGM) (10). 


\section{Study design and sample size determination}

142 A quantitative cross-sectional study complemented by qualitative study design was used 143 to determine dietary diversity practice and its influencing factors among pregnant women

144 from October 1 to November 10, 2018. The sample size for quantitative data was 145 calculated using a single proportion formula with the assumption of a small total 146 population (512). Thus, considering 95\% confidence level with a 5\% precision, and taking $14757 \%$ inadequate dietary diversity from a prior study in Dire Dawa, Eastern Ethiopia (7), 148 The sample size was found to be 217 . After adding for $15 \%$ non-response rate, the final 149 sample size was 250. Focus group discussions (FGDs) with groups of pregnant women 150 were conducted until the qualitative data was saturated.

\section{Inclusion and exclusion criteria}

152 All pregnant women who agreed to take part in the study and had lived in the study area 153 for at least one year prior to the study period were included. The study did not include 154 pregnant women who had chronic conditions like cancer or diabetes. This was due to the 155 fact that these illnesses are known to have an impact on a person's food consumption 156 and nutritional status. The respondents' health information was used to compile this data. 157 Pregnant women who had consumed special diets in the previous 24 hours owing to 158 holidays or celebrations were also excluded.

\section{Sampling technique and procedures}

160 The sampling interval was calculated by dividing the total number of pregnant women 161 who attended antenatal care in the health facility three months ago by the total sample 162 size. Then, using systematic sampling technique, the required study subjects were 
163 recruited. From the first two study subjects, the first study subject was selected by lottery, 164 and then every second study subject was selected until the required sample size was

165 achieved. But, pregnant women who had previously participated in the study did not re166 interview. Purposive sampling was used to select focus group discussants (FGD) for the 167 qualitative study. The FGD participants, on the other hand, were not the same as those 168 who were sampled for quantitative data.

\section{Data collection instruments and procedures}

170 For quantitative data: Data on demographic, socio-cultural, and economic factors, as well 171 as maternal health service utilization factors were constructed from previous literature 172 and collected by face-to-face interview using pre-tested structured questionnaires. Three 173 data collectors (two midwives and one nurse) were trained to collect quantitative data, 174 whereas two data collectors (two nurses) were recruited and trained to collect qualitative 175 data. The training allowed them to become familiar with the food groups and particular 176 foods within each food groups in order to place recalled foods into the appropriate food 177 groups. Data on dietary diversity was collected using a modified individual dietary 178 diversity Score (IDDS) questionnaire developed from food and agriculture organization 179 (FAO) (11). Individual dietary diversity Score (IDDS) questionnaire consisted of nine food 180 groups. All of the data collectors were fluent in Afar language (native). The questionnaires 181 are first written in English and then translated into the Afar language by a third person 182 who is a native Afar speaker with translation experience. Health extension workers in the 183 community and midwives at the Awash seven health centre's antenatal care (ANC) clinic 184 were also consulted to amend the food list in the food groups, whether or not they 185 matched with local names of foods, and acceptable terminology was agreed upon 
186 (modification was carried out based on local language). The respondents were asked to 187 recall all foods (meals and snacks) consumed the previous day and night (24-hour recall). 188 After recalling all foods and beverages consumed, these food items were recorded. The 189 interviewers underlined the corresponding foods in the list under the appropriate food 190 categories and entered " 1 " in the column next to the food group if at least one food was 191 consumed. Once the recall was completed, look for food groups that were not consumed 192 by respondents. After ensuring that no meals from that food group were eaten, "0: was 193 filled in the right-hand column corresponding to the food group.

194 Qualitative study: The qualitative data was collected by focus group discussion using 195 open-ended guiding question which was developed based on the study objective. The 196 purpose of study was explained for each study participant before data collection started 197 for both quantitative and qualitative data. Those who agreed to take part in the study were 198 then interviewed face-to-face in a quiet, comfortable and convenient location in order to 199 better understand each other and ensure confidentiality. The handwritten field notes from 200 all of the focus groups were transcribed into Microsoft Word 7, and everything was 201 translated from Afar to English, including certain key Afar words in brackets. On a daily 202 basis, the supervisor checked the collected data for completeness and accuracy. The 203 guiding questions used to interview focus group discussions was shown (Table 1).

204 Table 1: Shows the key questions used in focus group discussion interviewing guidelines.

Key questions 
Pregnant women

205

211 Good dietary diversity: Pregnant women who consumed four and above food groups out 212 of a total of nine food groups.

213 Poor dietary diversity: For those pregnant women who did not meet the minimum dietary

214 diversity requirement (consumption of less than four food group).

\section{Data analysis}

216 SPSS Version 23 was used for the analysis. Tables with frequency were used to present 217 the results. Binary logistics regression was used to find significant associations between 218 dietary diversity practice and independent variables. Variables having a p-value less than 2190.05 in binary logistic regression were included in multivariate logistic regression. The 
220 regression analysis' results were provided as odd ratios (OR), with $95 \% \mathrm{Cl}$ and a 221 significance level of less than 0.05 . When respondents consumed four or more food 222 groups, their responses were categorized as good, and if they consumed less than four 223 food groups, they were categorized as poor. The qualitative data was manually analysed 224 using the framework techniques in multidisciplinary research (12) The results of the 225 thematic analysis are presented in the form of narrative with supporting quotes. Finally, 226 the qualitative study's findings were triangulated with the quantitative findings.

\section{Ethical consideration}

228 The Ethics Committee at Samara University's Health Science College gave ethical 229 clearance. Before recruiting, informed consent was obtained from each mother after 230 explained about the study's objectives. Participation in the study was entirely voluntary. 231 All information was kept in strict confidence. 


\section{Quantitative study results}

242 Socio-demographic characteristics of pregnant women reside in Awash seven 243 district, Afar region of Ethiopia.

244 The response rate of the study was $96.4 \%$ (241/250). About $29.9 \%$ of participants were 245 in the age range of 21-25 years. Ninety-five percent of participants were married. Majority 246 of pregnant women (72.2\%) follows Muslim religious. More than half $(58.5 \%)$ of study 247 participants were Afar ethnicity. Almost three-fourth (75.9) of participants were urban 248 residents. Illiterate pregnant women represented 35.3\% of study participants. Moreover, 249 around three-fourth (75.5\%) of pregnant mothers were housewives. Twenty-seven 250 percent of pregnant women lived in extended family members (above four family 251 members). Nearly half $(50.6 \%)$ of study participants had average family income between 252 1500-3000 birr (Table 2).

253 Table 2: shows socio-demographic characteristics of pregnant women reside in Awash 254 seven district, Afar region of Ethiopia.

\begin{tabular}{lll}
\hline Variables & Categories & No (\%) \\
\hline Age & $=<20$ & $51(21.2)$ \\
& $21-25$ & $72(29.9)$ \\
& $26-30$ & $66(27.4)$
\end{tabular}


$>=31$

Marital status

Religious

Ethnicity

Residence

Educational status

Occupation
Married

Unmarried

Orthodox

Protestant and catholic

Muslim

Others

Amara

Afar

Urban

Rural

Illiterate

Primary school

Secondary school

College and above

Housewives

Government employed
$52(21.6)$

$229(95 \%)$

$12(5 \%)$

$55(22.8)$

$12(5)$

$174(72.2)$

39 (16.2)

$61(25.3)$

$141(58.5)$

$183(75.9)$

$58(24.1)$

85 (35.3)

68 (28.2)

$63(26.1)$

25 (10.4)

$182(75.5)$

$23(9.5)$ 
Merchants

Family size / members Four and below

Above four

Average monthly income $<1500$

(Ethiopian birr)
$36(14.9)$

$176(73 \%)$

$65(27 \%)$

$53(22)$

$122(50.6)$

$33(13.7)$

$33(13.7)$

255 Others represent Oromo, southern nation and nationalities peoples, and Tigray peoples

256 Obstetric and health service utilization characters of pregnant women reside in 257 Awash seven district, Afar region of Ethiopia.

258 About $67.3 \%$ of multiparous pregnant women had a birth interval of more than two years.

259 Nulliparous participants accounted for $32.4 \%$ of the total study participants. Almost half 260 of the study participants (49.8\%) were more than six months pregnant. Only $7.1 \%$ of study 261 participants had four antenatal care visit, while $49 \%$ of pregnant women had once 262 antenatal care visits (Table 3).

263 Table 3: Shows obstetric and health service utilization characters of pregnant women 264 reside in Awash seven district, Afar region of Ethiopia.

\begin{tabular}{lll}
\hline Variables & Categories & No (\%) \\
\hline Birth interval & Two year and below & $53(32.7)$
\end{tabular}




$\begin{array}{lll} & \text { Above two year } & 109(67.3) \\ \text { Parity } & \text { Nulliparous } & 78(32.4) \\ & \text { Multiparous } & 163(67.6) \\ \text { Month of pregnancy } & \text { Less than 3 months } & 38(15.8) \\ & 3-6 \text { months } & 83(34.4) \\ & \text { Greater than 6 months } & 120(49.8) \\ \text { Antenatal care visits } & \text { One } & 118(49) \\ & \text { 2-3 times } & 106(44) \\ & \text { Above three } & 17(7.1)\end{array}$

267 Ethiopia.

268 Individual dietary diversity scores of study participants had mean and standard deviations

269 of 3.1 and 1.38 , respectively. Twenty-seven percent of study participants had good dietary

270 diversity practice, whereas the remaining participants had poor dietary diversity practice.

271 Only $9.5 \%$ of pregnant women consumed meals five times a day, whereas $74.2 \%$ of study

272 participants ate three times a day. Nearly thirty-seven percent $(37.3 \%)$ of pregnant

273 women avoided certain types of meals during their pregnancy. During pregnancy, $58.9 \%$

274 and $13.3 \%$ of pregnant women avoided at least one of the protein foods (meat and eggs)

275 and semi-solid foods (cereal soup and porridge), respectively, out of the total food 276 restricted participants. (Table 4). 
277 Table 4: Dietary habit of pregnant women reside in Awash seven district, Afar region of 278 Ethiopia.

\begin{tabular}{lll}
\hline Variables & Categories & No (\%) \\
\multicolumn{2}{l}{ The mean of individual dietary diversity score (IDDS)= 3.1 } & \\
\hline Dietary diversity & Poor & $176(73)$ \\
& Good & $65(27)$ \\
Frequency of food taking per day & Three times & $178(74.2)$ \\
& Four times & $40(16.7)$ \\
Is there certain types of food taboos? & Five times & $22(9.2)$ \\
& Yes & $90(37.3)$ \\
& No & $151(62.7)$
\end{tabular}

Types of foods avoided during pregnancy Reason for prohibition

$\begin{array}{lll}\text { Protein (meat and egg) } \quad \text { Producing big fetus } & 53(58.9)\end{array}$

Semi-solid foods (porridge and cereal Attached to the fetus body 12 (13.3) made soup)

Milk

Producing Big fetus and 11(12.2)

attached to the fetus body

Fruit and vegetable (banana and cabbage) Attached to the fetus body

Carbohydrate (bread and other sweet Producing Big fetus foods) 
279 Pregnant women consumed food groups in the previous 24 hours in Awash seven district, Afar region of Ethiopia.

281 Over ninety percent of participants (91.3 percent) ate starchy staple foods (teff, wheat

282 and maize). About $61.8 \%$ of pregnant women consumed legumes, nuts and seeds. Milk 283 and milk production were taken by more than half of the participants (57.7\%) (Table5).

284 Table 5: The types of food groups consumed by pregnant women in the previous 24 hours 285 in Awash seven district, Afar region of Ethiopia.

Starchy Staple (cereal based like teff, wheat and maize)

Dark green leafy vegetables (kale and spinach)

other vitamin A rich foods and vegetables (mango, papaya and orange)

Other fruits and vegetables (avocado, lemon, cabbage, banana and apple

Organ meat

Meat and fish

Eggs

Legume, nuts and seeds

Milk and milk products
$220(91.3)$

$44(18.3)$

$61(25.3)$

$61(25.3)$

$41(17)$

$139(57.7)$ women reside in Awash seven district, Afar region of Ethiopia.

289 When confounding factors were adjusted, participants under the age of 20 years $290(\mathrm{AOR}=5.8$; at $95 \% \mathrm{Cl}: 1.6-13.5)$ and aged between $21-25$ years $(\mathrm{AOR}=3.9$; at $95 \% \mathrm{Cl}: 1.1-$ 291 12.2) had higher good dietary diversity consumption than those over the age of 30 years. 
292 Participants with a monthly average family income of less than 1500 birr (AOR=0.1; at 293 95\% Cl:0.02-0.7) and 1500-300 birr (AOR=0.05; at 95\% Cl: 0.01-0.2) had less dietary 294 diversity consumption than those with a monthly average family income of more than 2954500 birr. Pregnant women who had once antenatal care visit were 0.18 times more likely 296 to have a minimum dietary diversity consumption than those who had four or more 297 antenatal care visits (AOR=0.18; at 95\% Cl:0.04-0.8) (Table 6).

298 Table 6: Shows influencing factors associated with dietary diversity practices among 299 pregnant women reside in Awash seven district, Afar region of Ethiopia.

\begin{tabular}{|c|c|c|c|c|c|}
\hline \multirow[t]{3}{*}{ Variables } & & \multicolumn{2}{|c|}{ Dietary diversity } & \multirow{3}{*}{$\begin{array}{l}\text { COR with } \\
95 \% \mathrm{Cl}\end{array}$} & \multirow{3}{*}{$\begin{array}{l}\text { AOR with } \\
95 \% \mathrm{Cl}\end{array}$} \\
\hline & & \multirow{2}{*}{$\begin{array}{l}\text { Poor } \\
\text { No (\%) }\end{array}$} & \multirow{2}{*}{$\begin{array}{l}\text { Good } \\
\text { No (\%) }\end{array}$} & & \\
\hline & & & & & \\
\hline \multirow[t]{4}{*}{ Age } & $=<20$ & 31 (12.9) & $20(8.3)$ & $3.1(1.2-7.7)$ & $5.8(1.6-13.5)$ \\
\hline & $21-25$ & $49(20.3)$ & $23(9.5)$ & $2.2(0.9-5.4)$ & $3.9(1.1-12.2)$ \\
\hline & $26-30$ & $53(22)$ & $13(5.4)$ & $1.2(0.5-3.0)$ & $1.6(0.4-6.1)$ \\
\hline & $>=31$ & $43(17.8)$ & $9(3.7)$ & 1 & 1 \\
\hline \multirow[t]{3}{*}{ Religious } & Orthodox & $32(13.3)$ & $23(9.5)$ & $2.5(1.3-4.7)$ & \\
\hline & Protestant \& catholic & $9(3.7)$ & $3(1.2)$ & $1.2(0.3-4.5)$ & \\
\hline & Muslim & $135(56.2)$ & $39(16)$ & 1 & \\
\hline
\end{tabular}




\begin{tabular}{|c|c|c|c|c|c|}
\hline \multirow[t]{2}{*}{ Residence } & Urban & $125(52)$ & $58(24)$ & \multicolumn{2}{|l|}{$3.4(1.5-7.9)$} \\
\hline & Rural & $51(21.2)$ & $7(2.9)$ & 1 & \\
\hline \multirow[t]{4}{*}{ Educational } & Illiterate & $75(31.1)$ & $10(4.1)$ & $0.1(0.03-0.3)$ & \\
\hline & Primary school & $51(21.2)$ & $17(7.1)$ & $0.2(0.1-0.6)$ & \\
\hline & Secondary school & $40(16.6)$ & $23(9.5)$ & $0.4(0.1-1.0)$ & \\
\hline & College and above & $10(4.1)$ & $15(6.2)$ & 1 & \\
\hline \multirow[t]{3}{*}{ Occupation } & Housewives & $143(59.5)$ & $39(16)$ & $0.4(0.2-0.9)$ & \\
\hline & $\begin{array}{l}\text { Government } \\
\text { employed }\end{array}$ & $11(4.6)$ & $12(5)$ & $1.7(0.6-4.9)$ & \\
\hline & Merchants & $22(9.1)$ & $14(5.4)$ & 1 & \\
\hline \multirow{2}{*}{$\begin{array}{l}\text { Monthly } \\
\text { family }\end{array}$} & $<1500$ & $43(17.8)$ & $10(4.1)$ & $0.1(0.03-0.3)$ & $0.1(0.02-0.7)$ \\
\hline & $1500-3000$ & $107(44.4)$ & $15(6.2)$ & $0.1(0.02-0.1)$ & $0.05(0.01-0.2)$ \\
\hline \multirow[t]{2}{*}{ (ETB) } & $3001-4500$ & $17(7.1)$ & $16(6.6)$ & $0.3(0.1-0.9)$ & $0.6(0.1-2.4)$ \\
\hline & $>4500$ & $9(3.7)$ & $24(10)$ & 1 & 1 \\
\hline \multirow{3}{*}{$\begin{array}{l}\text { Antenatal } \\
\text { care visits }\end{array}$} & One & $99(41.1)$ & $19(7.9)$ & $0.17(0.1-0.5)$ & $0.18(0.04-0.8)$ \\
\hline & $2-3$ times & $69(29)$ & 37 (15) & $0.5(0.2-1.3)$ & $0.9(0.2-3.9)$ \\
\hline & Four and above & $8(3.3)$ & $9(3.7)$ & 1 & 1 \\
\hline
\end{tabular}




$\begin{array}{lllll}\begin{array}{l}\text { Frequency } \\ \text { of eating }\end{array} & \text { Three times } & 147(61) & 31(13) & \mathbf{0 . 1 2}(\mathbf{0 . 1}-\mathbf{0 . 3}) \\ \text { per day } & \text { Four times } & 21(8.8) & 19(7.9) & 0.5(0.2-1.5) \\ & \text { Five times } & 8(3.3) & 14(5.8) & 1 \\ & \text { Yes } & 79(32.8) & 11(4.6) & \mathbf{0 . 2 5}(\mathbf{0 . 1}-\mathbf{0 . 5}) \\ \text { Food } & & 97(40.2) & 54(22.4) & 1\end{array}$

302 In the qualitative study, a total of 38 pregnant women involving in six focus group

303 discussions, four of which were held with urban residents and two with rural inhabitants.

304 In urban residents, seven pregnant women participated in each of the two focus group 305 discussion and six study participants involved in the remaining each two focus group 306 discussion. In rural residents, each of the two focus group discussion had six participants. 307 The majority of participants (44.7\%) were between the ages of $21-30$ years, with the 308 remaining $15.8 \%$ and $39.5 \%$ were under and over the age of 20 years, respectively. About $30928.9 \%$ and $44.7 \%$ of participants were illiterate and in primary school, respectively, while $31018.4 \%$ and $7.9 \%$ of pregnant women were in secondary school, and college and above, 311 respectively. Nearly ninety percent (89.5\%) of pregnant mothers were multiparous. More 312 than half of the participants (55.3\%) were pregnant for 3-6 months, and $34.2 \%$ were 313 pregnant for over six months. Half of the study participants had one prenatal care follow314 up, while $42.1 \%$ had $2-3$ antenatal care follow-ups. In this qualitative study, dietary taboos 315 during pregnancy was investigated among pregnant women. According to focus group 
316 discussants, the most commonly avoided foods during pregnancy were semi-solid and

317 solid cereal-based foods, fruit and vegetables, animal products and soft drinks. A key

318 theme, food taboos, was developed using codes and categories (Table 7).

319 Table 7: Shows the development of a key theme, food taboos during pregnancy, using 320 codes and categories based on reports from pregnant women reside in Awash district, 321 Afar region of Ethiopia.
A major theme
Categories
Codes

Food taboos during pregnancy Semi-solid and solid foods Porridge, soups, bread

Fruits and vegetable Banana, cabbage

Animal products Milk, meat, eggs

Soft drink Coca cola, sprit

323 The following are the details of the qualitative study's participant interviews.

324 During pregnancy, the majority of the participants believed that semi-solid foods, as well 325 as some fruits and vegetables, should be avoided due to they thought these foods were 326 stick with the fetus's body. A 35-years-old women explained that "porridge, cereal soup 327 (locally known as atimt), banana and cabbages were not consumed during pregnancy 328 since they can cling with the fetus body" (a 35-years pregnant woman in FGD1).

329 The majority of the group members reflected their opinion that sweat foods and animal 330 products should not be consumed by pregnant women since the fetus would grow large 
331 and difficult to deliver. A 28-years-old pregnant women stated that "in our society, most 332 pregnant women do not consume meat, egg and milk since it is believed that these foods 333 produced big fetus and cause delivery to be delayed" (a 28-years pregnant woman in 334 FGD2).

335 Participants in the focus group discussion (FGD) also avoided milk, fruits and semi-solid 336 foods when pregnant. A pregnant 29-year-old woman expressed her belief that "yogurt, 337 bananas and porridge were adhering to the unborn fetus' body, resulting in an abnormal 338 child. Thus, I did not eat these foods" (a 29- years old pregnant woman in FGD3).

339 Almost all of the participants in the focus groups discussion said that protein and 340 carbohydrate diets were forbidden during pregnancy. This was explained by a 37-year341 old woman who stated that "I did not consume meat, eggs and bread because they 342 increased the weight of the fetus, caused prolonged labor and bleeding" (a 37-years old 343 pregnant woman in FGD4).

344 Coffee with milk and sugar was not allowed to take pregnant women to prevent fetal 345 obesity and facilitate delivery. A 39-year-old pregnant woman clarified this, "in Afar 346 culture, a mixture of coffee, milk and sugar (locally known as ashara) is not taking during 347 pregnancy since it is believed that it increased fetus weight and difficulty to easily deliver" 348 (a 39-years old pregnant woman in FGD5).

349 The majority of group respondents avoided soft drinks and semi-solid foods during their 350 pregnancy due to concerns about a thin baby bone caused by soft drinks and the 351 attachment of semi-solid foods to the fetus body. A 30-years-old woman noted that 352 "Coca-Cola and Sprite made the fetus bone thin, and porridge and cereal soup (locally 
353 called atmit) stick to the fetus body. So, we did not consume these food items" (a 30354 years old pregnant woman in FGD6).

355

356

357

358

359

360

361

362

363

364

365

366

367

368

369

370 


\section{Discussions}

372 Inadequate dietary diversity can harm both the mother and the fetus, with the effects on

373 the fetus perhaps persists to childhood. To promote maternal nutrition, health, and child

374 development, it is vital to recognize the dietary diversity practices of pregnant women (1).

375 There is no study on the dietary diversity among pregnant women in Afar region of

376 Ethiopia. Thus, identifying the magnitude of dietary diversity practice and its influencing

377 factors among pregnant women in pastoral region of Afar, is merit for policy and program

378 consideration. The average dietary diversity score in the study was $3.1 \pm(S D=1.38)$.

379 Furthermore, $73 \%$ of study participants exhibited poor dietary diversity. These findings

380 were higher than that of a study in Kenyan (4) and North East Ethiopia (7). The

381 discrepancy in dietary diversity scores could be attributable to the study area, where more

382 cultural practice might influence food intake during pregnancy, as opposed to study

383 settings in North East Ethiopia, where urban inhabitants could have more nutrient

384 diversity. According to the current survey, $37.3 \%$ of pregnant women avoided certain

385 types foods. This finding is consistent with the finding in Eastern Nigeria (3). According to

386 international food policy research institutions, there is a positive relationship between

387 dietary diversity and nutrient adequacy (diet meeting requirement of energy and essential

388 nutrients). Furthermore, according to the food and agricultural organization (FAO), the

389 individual dietary diversity score (IDDS) shows the nutrient adequacy of the diet and the

390 food groups analysed in this score place a greater focus on micronutrient consumption.

391 Therefore, lack of adequate dietary diversity in the majority of pregnant women in the

392 current study suggested nutrient deficiency, implying that the requirement for sufficient

393 energy and key nutrients was not met. This evidence supports prior study in Wondo Genet 
district of Southern Ethiopia, which found that most pregnant women's nutritional intakes were below recommended levels (6). The majority of the participants in this study (91.3 \%) ate cereal-based diets. This result is consistent with a research in low and middleincome countries (2), but lower than a study in Kenya (4) that found cereal-based foods were the most popular (99\%). Furthermore, all food groups consumed by pregnant women in the current study were lower than in a study done in north east Ethiopia (7), with the exception of milk and milk products, which were common in the pastoral region of Afar. Meat and eggs were the most avoided foods during pregnancy and the least consumed food groups out of nine food groups. These findings were strengthening the qualitative finding, which revealed that animal products (meat and eggs) were the most popular avoided foods during pregnancy. Moreover, Semi-solid foods (porridge and cereal soup), milk, some fruits and vegetables, and carbohydrate-rich foods (bread and sweet foods) were also forbidden during pregnancy, according to study participants in both quantitative and qualitative investigations. Fear of fetus weight increase, which is linked to delivery difficulty, and the assumption that semi-solid meals, fruit, and vegetable attachments to the fetus body were the reasons for avoiding eating these foods. These findings are consistent with those of a prior study conducted in Eastern Nigeria (3), Ethiopia's Oromia area (5) and Afar region Ethiopia (8). Participants under the age of 20 years and those between the ages of 21-25 years consumed better dietary diversity than those over the age of 30 years. This could be due to the fact that older women are more likely to adhere to food taboos. This evidence is related with a study in Oromia region of Ethiopia, which found that elderly women were more likely to practice dietary taboos than younger women (5). Pregnant women with a low monthly average family income (under 
4173000 birr) were less likely than those with a higher average monthly family income (over

4184500 birr) to practice the minimal dietary diversity. This finding is similar with a study

419 finding in Kenya (4). Pregnant women who had once antenatal care visit were also less

420 likely to consume the minimum dietary diversity than those who had four or more. The

421 minimum dietary diversity did not have a significant association with educational level and

422 occupation. This is contradicting with a study in Kenya (4). This could be more antenatal

423 care visits are linked to increased awareness of the benefits of nutritional diversity.

424 This study is cross-sectional study, so, it is difficult to infer a causal association. Adding 425 an exploratory study with a quantitative study, on the other hand, aids in the discovery of 426 additional information not available from the quantitative study. The open-ended 427 questionnaire allowed participants to report any foods or beverages they had consumed 428 or not consumed without restriction. Thus, these questionnaires allowed women to 429 describe their experiences in their own words, but they were vulnerable to social 430 desirability. Using a single 24-hour recall period did not reveal an individual's habitual 431 diet, and the amount of food consumed is not indicated by the dietary diversity score. 432 Attainment of pregnant women at antenatal clinics was low, particularly in rural areas, 433 resulting in a smaller number of participants being recruited from these areas. The fact 434 that the sample was limited to a single season may restrict the generalizability of the 435 results to subsequent seasons. However, the study provided a new perspective on factors 436 that influence dietary diversity among pregnant women, particularly in the pastoral 437 community of Afar, which is novel and interesting. Therefore, the findings can give useful 438 information for nutrition-sensitive intervention. 
439 Conclusion: The majority of study participants consumed below the minimum dietary 440 diversity. Younger pregnant women had good dietary diversity than elder pregnant

441 women. Having a high average family income was associated with good dietary diversity.

442 Those pregnant women with once antenatal care visits had less minimum dietary diversity

443 than those who had four or more antenatal care visits. Protein-rich foods (meat and eggs),

444 semisolid foods (porridge and cereal soup) and milk were the most commonly avoided 445 foods by pregnant women. The most common reason for avoiding these meals was 446 delivery difficulties and the fear that they might stick to the fetus's body.

447 List of abbreviation: FGD: focus group discussion, SPSS: social science statistical 448 package, COR: crude odd ration, AOR: adjusted odd ratio, CI: confidence interval, SD: 449 standard deviation.

\section{Declaration}

451 Ethical approval and consent to participate: Ethical clearance was obtained from a 452 research and ethics review committee of health science college, Samara University. All 453 methods were performed in accordance with the relevant guidelines and regulations. 454 Informed consent was obtained from each study participant after explanation of the 455 study's aim. No one was harmed as a result of participating in this study. By eliminating 456 any identifier from questionnaires, confidentiality was respected.

457 Consent for publication: Not applicable

458 Availability of data and material: Based on author request.

459 Competing interest: The author declared that no conflict of interest for these work. 
460 Funding: No funding source

461 Acknowledgement: The authors like to thank all study participants and other individuals

462 involving in any process of this study.

463 Author contributions: Conceive and design the study: T.G.W, Perform the study:

464 T.G.W, Analysis the data: T.G.W, Wrote the paper: T.G.W.

465

466

467

468

469

470

471

472

473

474

475

476

477 
479 1. Nnam N. Improving maternal nutrition for better pregnancy outcomes. Proceedings 480 of the Nutrition Society. $2015 ; 74(4): 454-9$.

481 2. Lee SE, Talegawkar SA, Merialdi M, Caulfield LE. Dietary intakes of women during 482 pregnancy in low-and middle-income countries. Public health nutrition. 2013;16(8):134048353.

484 3. Ekwochi U, Osuorah CD, Ndu IK, Ifediora C, Asinobi IN, Eke CB. Food taboos and 485 myths in South Eastern Nigeria: The belief and practice of mothers in the region. Journal 486 of ethnobiology and ethnomedicine. 2016;12(1):1-6.

4874 Kiboi W, Kimiywe J, Chege P. Determinants of dietary diversity among pregnant 488 women in Laikipia County, Kenya: a cross-sectional study. BMC Nutrition. 2017;3(1):1-8.

489 5. Zerfu TA, Umeta M, Baye K. Dietary habits, food taboos, and perceptions towards 490 weight gain during pregnancy in Arsi, rural central Ethiopia: a qualitative cross-sectional 491 study. Journal of Health, Population and Nutrition. 2016;35(1):1-7.

492 6. Kuche D, Singh P, Moges D, Belachew T. Nutritional status and associated factors 493 among pregnant women in Wondo Genet District, Southern Ethiopia. Journal of Food 494 Science and Engineering. 2015;5(2):85-94.

4957 Shenka A, Damena M, Abdo M, Roba KT. Dietary diversity and nutritional status 496 of pregnant women attending public hospitals in dire dawa city administration, eastern 497 Ethiopia. East African Journal of Health and Biomedical Sciences. 2018;2(1):10-7.

498 8. Hadush Z, Birhanu Z, Chaka M, Gebreyesus H. Foods tabooed for pregnant 499 women in Abala district of Afar region, Ethiopia: an inductive qualitative study. BMC 500 nutrition. 2017;3(1):1-9. 
501 9. Reda KT. Social organization and cultural institutions of the Afar of Northern 502 Ethiopia. International Journal of Sociology and Anthropology. 2011;3(11):423-9.

503 10. Andarge MY. The Difficulties of Ending Female Genital Mutilation (FGM): Case of 504 Afar Pastoralist Communities in Ethiopia. Retrieved fromwww ohchr 505 org/Documents/lssues/Women/WRGS/FGM/NGOs/ActionFor. 2014.

506 11. Kennedy G, Ballard T, Dop MC. Guidelines for measuring household and individual 507 dietary diversity: Food and Agriculture Organization of the United Nations; 2011.

508 12. Gale NK, Heath G, Cameron E, Rashid S, Redwood S. Using the framework 509 method for the analysis of qualitative data in multi-disciplinary health research. BMC 510 medical research methodology. 2013;13(1):1-8. 